\title{
Analysis of the Disintegrin-metalloproteinases Family Reveals ADAM29 and ADAM7 Are Often Mutated in Melanoma
}

\author{
Xiaomu Wei ${ }^{1}$, Angela Moncada-Pazos ${ }^{2}$, Santiago Cal $^{2}$, Clara Soria-Valles ${ }^{2}$, Jared Gartner ${ }^{1}$, \\ Udo Rudloff ${ }^{3}$, Jimmy C. Lin ${ }^{4}$, NISC Comparative Sequencing Program ${ }^{5}$, Steven A. \\ Rosenberg ${ }^{3}$, Carlos López-Otín ${ }^{2}$, and Yardena Samuels ${ }^{1, *}$ \\ ${ }^{1}$ Cancer Genetics Branch, National Human Genome Research Institute, National Institutes of \\ Health (NIH), Bethesda, Maryland, 20892, USA \\ ${ }^{2}$ Departamento de Bioquímica y Biología Molecular, Instituto Universitario de Oncología (IUOPA), \\ Universidad de Oviedo, 33006-Oviedo, Spain \\ ${ }^{3}$ Surgery Branch, National Cancer Institute, NIH, Bethesda, Maryland 20892, USA \\ ${ }^{4}$ Ludwig Center for Cancer Genetics and Therapeutics, and Howard Hughes Medical Institute at \\ the Johns Hopkins Kimmel Cancer Center, Baltimore, Maryland 21231, USA \\ ${ }^{5} \mathrm{NIH}$ Intramural Sequencing Center, National Human Genome Research Institute, National \\ Institutes of Health, Bethesda, Maryland, USA
}

\begin{abstract}
We performed a mutational analysis of the 19 disintegrin-metalloproteinases (ADAMs)genes in human cutaneous metastatic melanoma and identified eight to be somatically mutated in 79 samples, affecting $34 \%$ of the melanoma tumors analyzed. Functional analysis of the two frequently mutated ADAM genes, ADAM29 and ADAM7 demonstrated that the mutations affect adhesion of melanoma cells to specific extracellular matrix proteins and in some cases increase their migration ability. This suggests that mutated ADAM genes could play a role in melanoma progression.
\end{abstract}

\section{Keywords}

Somatic mutation; Melanoma; ADAM7; ADAM29

\section{INTRODUCTION}

The matrix metalloproteinase (MMP) proteolytic enzymes have recently been shown to be frequently mutated in melanoma (Palavalli, et al., 2009). The MMPs are only one family within a superfamily of zinc-based proteinases, the metzincins (Stocker and Bode, 1995). Another family of metzincins is the ADAMs (a disintegrin and metalloproteinase) which are membrane anchored glycoproteins with several biological functions encompassing cell adhesion, cell fusion and signaling. About half of the ADAMs have a consensus metalloproteinase catalytic sequence, giving them proteolytic activity, whereas the rest have cell adhesion roles. Well characterized ADAM family members include ADAM17 (TACE) which causes ectodomain shedding of various membrane-associated molecules including TNF- $\alpha$ and EGF-related ligands (Peschon, et al., 1998), as well as ADAM10 which has been

(C) 2011 Wiley-Liss, Inc.

*Correspondence to Yardena Samuels; samuelsy@mail.nih.gov.. 
shown to act via the Notch pathway (Qi, et al., 1999) and through shedding of ephrins (Hattori, et al., 2000). Although the function of some of the ADAMs has been characterized, the function of most family members and their involvement in cancer is as yet unknown (Blobel, 2005; Schlondorff and Blobel, 1999).

Several studies suggest a direct role of ADAM family in human cancer development and progression. For example, ADAM17 is an upstream regulator of the EGF signaling pathway, one of the most commonly altered signal transduction pathways in cancer. Additionally, the presence of distinct somatic mutations in several members of the ADAM family further suggests a direct involvement of these genes in cancer genesis (Dalgliesh, et al., 2010; Parsons, et al., 2008; Pleasance, et al., 2010; Sjoblom, et al., 2006; TCGA, 2008). However, previous reports investigating the extent of ADAM mutations in cancer are incomplete as they either did not investigate the entire ADAM gene family or were limited to particular cancer types (Sjoblom, et al., 2006; Wood, et al., 2007).

In this study, we systematically analyzed the entire ADAM gene family in a large panel of human melanomas. Melanoma is the most common fatal skin cancer and despite years of research, metastatic disease has a dismal prognosis. The median patient survival is six months following diagnosis of late-stage disease, with fewer than 5\% surviving five years (Jemal, et al., 2009). Identification of genes affected by somatic mutations in metastatic melanomas should provide new opportunities for clinical intervention. Our comprehensive genetic study identified two frequently mutated ADAM genes, ADAM29 (MIM\# 604778) and ADAM7 (MIM\# 607310).

Functional analysis of a subset of mutations identified in ADAM29 showed them to increase their adhesion to collagen I, II and IV. In contrast, analysis of ADAM7 mutations showed reduced cell adhesion to collagen IV and laminin and increased melanoma cell migration. Our study is the first comprehensive mutational analysis of the ADAM family in human cancer and provides evidence for the association of ADAM in melanoma tumorigenesis.

\section{MATERIALS AND METHODS}

\section{Tumor Tissues}

A panel of pathology-confirmed metastatic melanoma tumor resections, paired with apheresis-collected peripheral blood mononuclear cells, was collected from 79 patients enrolled in IRB-approved clinical trials at the Surgery Branch of the National Cancer Institute. Pathology-confirmed melanoma cell lines were derived from mechanically or enzymatically dispersed tumor cells, which were subsequently cultured in RPMI $1640+$ $10 \% \mathrm{FBS}$ at $37^{\circ} \mathrm{C}$ in $5 \% \mathrm{CO}_{2}$ for 5-15 passages. Genomic DNA was isolated using DNeasy Blood \& Tissue kit (Qiagen, Valencia, CA).

\section{PCR, sequencing and mutational analysis of melanoma samples}

PCR and sequencing was done as previously described (Palavalli, et al., 2009; Prickett, et al., 2009; Viloria, et al., 2009). The primary phase mutation screen was analyzed using Consed (Gordon, et al., 1998). Variants were called using Polyphred 6.11 (Bhangale, et al., 2006) and DIPDetector (Hansen N., unpublished), an indel detector for improved sensitivity in finding insertions and deletions. Sequence traces of the secondary screen were analyzed using the Mutation Surveyor software package (SoftGenetics, State College, PA).

In this study all coding exons of the ADAM gene superfamily in 31 melanoma patients were included into the screen and a total of 401 exons from the ADAM were extracted from genomic databases (Supp. Table S1). These exons as well as at least 15 intronic bases at both the 5 ' and 3 ' ends including the splicing donor and acceptor sites, were amplified by 
using polymerase chain reaction (PCR) from cancer genomic DNA samples using the primers listed in Supp. Table S2. Out of the 367 exons amplified, 96\% were successfully sequenced using dye terminator chemistry.

Validation of somatically mutated genes was performed in an additional 48 tumor samples. A total of 20,098 PCR products, spanning 7Mb of tumor genomic DNA, were generated and sequenced. Sequence data for each amplicon were evaluated for quality within the target region. To avoid PCR or sequencing artifacts we re-amplified and re-sequenced amplicons that had alterations.

The DNA mutation numbering system used in this study was based on cDNA sequence. Nucleotide numbering reflects cDNA numbering with +1 corresponding to the $\mathrm{A}$ of the ATG translation initiation codon in the reference sequence, according to journal guidelines (www.hgvs.org/mutnomen). The initiation codon is codon 1.

\section{Construction of wild-type and mutant ADAM29 and ADAM7 expression vector}

Human ADAM7 (NM_003817.2) and ADAM29 (NM_014269.4) were purchased from Open Biosystems and PCR cloned into the mammalian expression vector pCDF-MCS2-EF1Puro $^{\mathrm{TM}}$ (Systems Biosciences, Inc., Mountain View, CA) or pCDNA3.1 (-) (Invitrogen, Molecular Probes) via the XbaI and NotI restriction sites. Flag tag was introduced into 3' end of the gene. Cloning primers for $A D A M 7$ are: 5' -

GCTGTCTAGAGCCACCATGAAGATGTTACTCCTGCTGCATTGCCTTGGG - 3' and 5' - ATCAGCGGCCGCCTACTTATCGTCGTCATCCTTGTAATCCTTGGCACTTTG -

3'. Cloning primers for ADAM29 are 5'-

GCTGTCTAGAGCCACCATGAAGATGTTACTCCTGCTGCATTGCCTTGGGG - 3 ' and 5'- ATCAGCGGCCGCCTACTTATCGTCGTCATCCTTGTAATCGGAGGGCGTCA - 3'. The ADAM7 and ADAM29 mutants were generated using Phusion PCR for site-directed mutagenesis.

\section{Generation of Mel-STR and A375 stable pooled clones}

Mel-STR cells were maintained in RPMI-1640 supplemented with 10\% FBS. Cells were seeded in T175 flasks at 70\% confluent the day before transfection. pcDNA3.1(-) empty vector or ADAM7 (WT, p.H243Y, p.M359I, p.E639K, p.S703N) were transfected into cells using Fugene6 (Roche, Indianapolis, IN) following manufacturers protocol. $48 \mathrm{hrs}$ after transfection, cells were selected using normal growth medium supplemented with $300 \mu \mathrm{g} / \mathrm{ml}$ G418.

To make lentivirus for ADAM29 A375 stable pooled clones, pCDF-ADAM29 (WT, p.E111K, p. S112F, p.S115F, p.E176K, p.I257F, p.G434D, p.E503K) constructs were cotransfected into HEK 293T cells seeded at $1.5 \times 10^{6}$ per T75 flask with pVSV-G and pFIV-34N (kind gifts from Todd Waldman, Georgetown University) helper plasmids using Fugene6 (Roche, Indianapolis, IN). Virus was harvested $48 \mathrm{hrs}$ after transfection. A375 cells were seeded at $1.5 \times 10^{6}$ cells per T75 flask $24 \mathrm{hr}$ prior to infection. $24 \mathrm{hrs}$ after infection, cells were selected using normal growth medium supplemented with $3 \mathrm{ng} / \mathrm{ml}$ puromycin.

\section{Proliferation assay}

Mel-STR or A375 cells were seeded in 96 well plates at 250 cells per well in normal growth medium and incubated for 13-17 days. Proliferation of cells were monitored every 48 hrs. Cells were incubated in $50 \mu 10.2 \%$ SDS/well at $37^{\circ} \mathrm{C}$ for $2 \mathrm{hrs} .150 \mu \mathrm{l} /$ well SYBR Green I solution (1:750 SYBR Green I (Invitrogen-Molecular Probes) diluted in $\mathrm{dH}_{2} 0$ ) were added to cells before analyzing using a BMG Labtech FLOUstar Optima. 


\section{Reverse Transcription PCR}

Total RNA was extracted from melanocytes, melanoma cells and pooled clones following the manufacturer's protocol for RNeasy Mini Kit (Qiagen \#74101). Total RNA was eluted in $30 \mu \mathrm{l}$ DEPC-treated dH20. A total of $1 \mu \mathrm{g}$ of total RNA was used for single strand cDNA synthesis using a SuperScript III First Strand kit (Invitrogen \#18080-051). cDNA was amplified using the oligo dT20 primer supplied in the kit. PCR primers used for ADAM7 message are 5'-ACACGGAAGGATTTTGATCATGTTG - 3' and 5'GGATTGGCTCAGTCCTTATCTGCTG - 3'., ADAM29 PCR primers are 5'GATCTGGACCAATAAAAACCTCATTGTAGTAGATGATGTAAGGAA -3' and 5'CATTTTTGATACCACAGTGACCAACACGGTCACCTAAGG - 3'. GAPDH primers (forward primer: 5'- TGGAAGGACTCATGACCACA - 3', reverse primer: 5'TGCTGTAGCCAAATTCGTTG - $3^{\prime}$ ) were used as a control. The product was then analyzed on a $1 \%$ agarose gel.

\section{Adhesion Assay}

Adhesion capacity of different cell pools was analyzed with the ECM Cell Adhesion Array kit (Colorimetric) (EMD Biosciences, ECM540 96 wells) following manufacturer's instructions.

\section{Migration assays}

Mel-STR ADAM7 stable clones were seeded into migration wells $(8.0 \mu \mathrm{m}-\mathrm{BD}$ Biocoat, BD Biosciences) at 10,000 cells per well in serum-free medium in top chambers and incubated for $24 \mathrm{hrs}$. The bottom chamber contain normal growth medium. Migrated cells were fixed and stained using Hema 3 Stat Pack.

\section{RESULTS}

The human ADAM family consists of 19 genes (Supp. Table S1). While previous studies listed in Supp. Table S3 investigated the ADAM gene family mutational status, results are not comprehensive. As a few of the ADAM genes were found to be altered in melanoma previously, we decided to systematically evaluate the mutation status of all the gene family members in a large panel of cutaneous metastatic melanoma samples. The coding exons of the ADAMs gene superfamily were genetically evaluated in 31 melanoma patients. To determine whether a mutation was somatic (i.e., tumor specific) we examined the sequence of the gene in genomic DNA from normal tissue (derived from blood) of the relevant patient. This allowed the identification of eight genes containing somatic mutations. Genes found to have one non-synonymous mutation or more were then further analyzed for mutations in an additional 48 melanomas. Through this approach, we identified 41 mutations in eight genes, thus affecting $34 \%$ of the melanoma tumors analyzed (Table 1).

The observed somatic mutations could either be "driver" mutations, on which tumor growth is dependent, or "passenger" events that confer no selective advantage to tumor growth. In the eight genes found to be mutated, 41 non-synonymous and 10 synonymous somatic mutations were identified, yielding a N:S (non-synonymous: synonymous) ratio of 4.1:1, a value significantly higher than the $\mathrm{N}: \mathrm{S}$ ratio of $2: 1$ predicted for nonselected passenger mutations $(p<0.03)$ (Sjoblom, et al., 2006), suggesting that many of these are likely to be "driver" mutations. Most somatic mutations base substitutions in our screen were $\mathrm{C}>\mathrm{T} / \mathrm{G}>\mathrm{A}$ transitions and were significantly greater than other nucleotide substitutions $(p<0.002)$ (Supp. Figure S1), which is reminiscent of the mutation pattern reported previously caused by ultraviolet light exposure (Greenman, et al., 2007). 
One of the most frequently mutated genes, $A D A M 7$, harbored two alterations in the same residue (p.G302E), forming a mini-hotspot. Evaluation of the mutation status of $A D A M 7$ in commercially available melanoma cell lines revealed two additional mini-hotspots. The mutation p.H243Y found in the SK-Mel5 melanoma line was also present in the 72T sample, and the melanoma cell line SK-Mel28 was found to harbor the p.M359I mutation which was also detected in the $23 \mathrm{~T}$ melanoma sample. The three mini-hotspots occur within the same functional reprolysin domain (Supp. Figures S2 and S3A).

Eight of the genes found to be mutated in our melanoma panel were previously found to be altered in several cancer types (highlighted in Supp. Table S3). Only four of these were found to be mutated in melanoma. Notably, seven of the previously observed alterations lie close to the mutations detected in this study. ADAM18 which was formerly found to be mutated on residue p.T583I in glioma was mutated on residue p.S536 in our melanoma panel. ADAM28 which was previously reported to harbor a mutation in residue $\mathrm{p}, \mathrm{D} 470 \mathrm{~N}$ in melanoma was identified to harbor two neighboring somatic mutations (p.G450E and p.S482F) in our melanoma panel. ADAM19 which was reported to contain the p.L425V alteration in the disintegrin domain had a p.Q500X alteration in the same domain in our studies. Finally, the most frequently mutated gene, ADAM29, was previously found to be mutated in melanoma in the recently published whole genome report by the Sanger Center (Pleasance, et al., 2010). Importantly, all previously identified mutations (p.V205I, p.C534X and p.G589E) lie in the same domains as our newly discovered mutations (Table 2 and Supp. Figure S3).

The clinical information associated with the melanoma tumors containing somatic ADAM mutations is provided in Supp. Tables S4 and S5. There was no association of the detected mutation pattern with any of the analyzed clinical or pathological characteristics of the melanoma patients.

$A D A M 7$ was one of the most frequently mutated ADAM genes, as it was found to be somatically mutated in over $12 \%$ of melanoma cases (Table 1 ). In addition, taking into account a background mutation rate in melanoma of $11 \mathrm{mut} / \mathrm{Mb}$ (paper under review), the observed mutations in $A D A M 7$ are significantly different to that expected by chance ( $\mathrm{p}=$ 1.32E-06). The alterations identified in ADAM7 occurred in residues that are highly conserved evolutionarily, retaining identity in rat and mouse (Supp. Figure S4).

Furthermore, the identified somatic mutations clustered onto various important functional domains of the ADAM family (Supp. Figure S3A). While mutations p.P14S, p.R31C, p.P36S and p.H106Y all occur in the propeptide domain. Mutations p.H243Y, p.G302E and p.M359I are within the reprolysin domain. To provide a computational estimation of the effects of the different missense mutations, we used the SIFT (sorting intolerant from tolerant) algorithm (Ng and Henikoff, 2003). This data is presented in Supp. Table S6 and shows that at least six of the eleven alterations would affect protein function. Interestingly, in contrast to melanoma cells, no $A D A M 7$ was found to be expressed in human melanocytes (Supp. Figure S5). The combination of this data suggests that mutant $A D A M 7$ is likely to function as a driver in melanoma.

Based on the potential functional relevance of the altered residues in ADAM7, and the fact that ADAM7 does not have protease activity we chose to clone the two cytoplasmic mutations p.E639K and p.S703N as well as the p.H243Y and p.M359I mutations for further studies. To test the effects of these mutations on ADAM7 function we created stable pooled clones in human melanoma Mel-STR cells (Gupta, et al., 2005) which harbor wild-type $A D A M 7$ (Supp. Table S7). As seen in Supp. Figure S6A the cell clones expressed similar levels of wild-type or mutant ADAM7. These clones were then used for further studies. We first observed that expression of wild-type or mutant ADAM7 did not affect the growth rate 
of Mel-STR cells in tissue culture (Supp. Figure S6B). Then, and because ADAM family members are known to modulate tumor cell adhesion by interactions with proteins in the basal lamina (Edwards, et al., 2008), we evaluated whether the mutations in ADAM7 affected its adhesion to different extracellular matrix components. For this purpose, we used the above described Mel-STR pooled clones expressing wild-type or mutant ADAM7 in an adhesion assay. This assay revealed that mutant ADAM7 conferred significantly reduced binding on collagen IV (p.M359I, p.E639K and p.S703N) and laminin-1 (p.H243Y, p.E639K and p.S703N) compared with the wild-type expressing cells $(P<0.005, t$-test $)$ (Figure 1).

As previous studies reported that reduced adhesion facilitates cell migration (Touab, et al., 2002; Yamagata, et al., 1989), our finding that cells expressing mutant ADAM7 have reduced collagen IV and laminin-1 adhesion prompted us to investigate whether these cells also have increased migration ability. As can be seen in Figure 2A, Mel-STR control cells migrated through the porous membrane. Interestingly, cells expressing wild-type ADAM7 resulted in $>60 \%$ reduction in migrating cells $(171+/-6.6$ versus $70+/-17.7)$. When the ADAM7 mutant expressing cells were tested in the same assay, two mutations (p.E639K and p.S703N) increased the cell migration capabilities compared to wild-type ADAM7 $(122+/-18.5$ and $210+/-33.6 P<0.001, t$-test $)$. Based on these results we can conclude that wild-type ADAM7 inhibits cell migration and that some ADAM7 mutations alleviate this, which might suggest that these mutations might be causing a loss of function effect.

To further our functional analysis of ADAM gene somatic alterations, we next focused on ADAM29 which was found to be somatically mutated in over $15 \%$ of melanoma cases (Table 1) making it the most highly mutated ADAM in this screen. Several criteria suggest $A D A M 29$ to also be a driver in melanoma (1) ADAM29 has previously been shown to harbor somatic mutations in other cancer types (Table 2), (2) the observed mutations in ADAM29 are significantly higher to that expected by chance $(\mathrm{p}=7.73 \mathrm{E}-08)$ (3) 14 non-synonymous $(\mathrm{N})$ and 0 synonymous (S) somatic mutations were identified in ADAM29, yielding a $\mathrm{N}: \mathrm{S}$ ratio of 14:0, which is significantly higher than the N:S ratio of 2:1 predicted for nonselected passenger mutations (Sjoblom, et al., 2006) (P < 0.003) and (4) SIFT analysis shows that seven of the 14 alterations in ADAM29 would affect its protein function (Supp. Table S8).

On this basis, we chose to functionally evaluate some of the mutations identified in ADAM29 as well. Somatic mutations in ADAM29 occurred mainly in the propeptide and reprolysin domains (Supp. Figure S3B). As the reprolysin domain encodes the catalytic portion of the protein and ADAM29 does not have protease activity, we decided to mainly focus on the mutations found in the propeptide domain especially as an interesting cluster of mutations was observed in this domain involving the p.E111K; p.S112F and p.S115F alterations (Supp. Figure S3B).

To determine whether mutations in ADAM29 affected cell growth, we created stable pooled clones expressing wild-type ADAM29 and seven tumor derived mutants (p.E111K, p.S112F, p.S115F, p.E176K, p.I257F, p.G434D and p.E503K) in A375 cells, which were shown to harbor wild-type ADAM29 (Supp. Table S9). A similar expression level of the ADAM29 constructs was observed (Supp. Figure S7A). Expression of wild-type or mutant ADAM29 did not affect the growth rate of A375 cells in tissue culture (Supp. Figure S7B). In contrast, expression of the various ADAM29 mutants substantially increased cell adhesion to collagen I and IV compared to cells expressing wild-type ADAM29 $(P<0.005$, $t$-test (Figure 3). Furthermore, five of the ADAM29 mutants (p.S112E, p.S115F, p.I257F, p.G434D and p.E503K) showed significantly increased adhesion compared to wild type ADAM29 to collagen II $(P<0.005, t$-test (Figure 3$)$. Binding to other extracellular matrix 
proteins such as fibronectin, laminin, tenascin and vitronectin showed some differential binding between wild type and some of the ADAM29 mutants, but this was not as striking as the binding to collagen I, II and IV (Supp. Figure S8).

\section{DISCUSSION}

We have performed the first systematic mutational analysis of the ADAMs gene family in any human cancer type leading to the discovery of two frequently mutated ADAM genes, $A D A M 7$ and ADAM29. The somatic mutations identified in this study were confirmed by multiple PCR and sequencing reactions. The mutations are novel and multiple mini-hotspot alterations were identified in ADAM7. The changes in ADAM7 and ADAM29 affect highly conserved residues. Our in silico analysis predicted that some of the mutated residues in ADAM7 and ADAM29 would affect tumorigenic phenotypes of melanoma cells. This was experimentally tested by cloning the relevant nucleotide alterations into ADAM7 as well as ADAM29 cDNA. Functional assays confirmed that four of the mutations in ADAM7 (p.H243Y, p.M359I, p.E639K and p.S703N) have altering effects on the wild-type protein function. Specifically, although cells expressing wild-type ADAM7 adhere to collagen IV and laminin, the four mutated proteins reduce this ability significantly. Intriguingly, in contrast to ADAM7, seven of the ADAM29 alterations (p.E111K, p.S112F, p.S115F, p.E176K, p.I257F, p.G434D and p.E503K) increase the adhesion of melanoma cells to collagen I and collagen IV, compared to wild-type ADAM29. These results emphasize the need to test the role of each ADAM and its related mutations in an individual manner to precisely define its functional role in cancer.

ADAM enzymes are zinc endopeptidases and contain a conserved zinc binding motif within the reprolysin family zinc metallopeptidase domain (PF01421). Of all the presumed functional human ADAM genes, 13 encode proteins that possess the characteristic reprolysin-type active site (HEXGHXXGXXHD) in the metalloproteinase domain followed downstream by the "Met turn" a key signature of metzincin enzymes (Bode, et al., 1993), indicating functional proteolytic capability (Edwards, et al., 2008). Several ADAM family members (including ADAM2, ADAM11, ADAM18, ADAM23 and ADAM32) lack all three conserved histidines found within the zinc-binding motif which prevents zinc binding and proteolytic activity. ADAM7 contains the "Met turn" as well as the three conserved histidines, but is missing a glutamic acid in the second position of the motif, which is one of the critical features in the Zn-binding active site (Supp. Figure S9). This suggests that the metalloproteinase domain in ADAM7 may play roles in protein folding and protein-protein interactions rather than cleaving components of extracellular matrix (Lopez-Otin and Bond, 2008). Interestingly, one of the ADAM7 mini-hotspot alterations discovered in our study occurs at the Met-Turn (p.M359I), which as mentioned above is highly conserved throughout the ADAM gene family, suggesting that this mutation would have substantial functional effects on the protein.

A role in signal transduction of this ADAM gene is further supported by the fact that the cytoplasmic domain of ADAM7 includes a phosphorylation site and a Src homology region 3 (SH3) binding domain. In particular, the motif LKQVQSP is recognized by those SH3 domains with non-canonical class II recognition specificity and the motif QVQSPPT is predicted as a proline-directed kinase phosphorylation site in higher eukaryotes.

Interestingly, the p.S703N mutation is found in this location and might therefore be involved in signalling.

Indeed, many ADAM cytoplasmic domains contain serine and proline residues, with ADAM7 containing the consensus class I (RXXPXXP) and class II (PXXPXR) ligands for interaction with SH3 domains of various intracellular proteins (Lopez-Otin and Hunter, 
2010; Seals and Courtneidge, 2003). These motifs also exist in other ADAM family members such as ADAM1, ADAM15 and ADAM17 but their positions as well as the neighboring sequences are different among the diverse ADAMs. It is therefore possible that each ADAM could form a unique complex of cytoplasmic proteins through specific proteinprotein interactions.

Bioinformatics analysis of the ADAM7 and ADAM29 mutations was performed based on sequence homology to other ADAM proteins in the family or in other species (Supp. Figure S10) (Ruan, et al., 2008). Both the p.E639K and p.S703N mutations in ADAM7 were outside of known existing protein family domains that were present in ADAM7 and were not significantly evolutionarily conserved ( $\mathrm{Ng}$ and Henikoff, 2003). It must be noted that domains, such as the Reprolysin, ADAM_CR, Pep_M12B_propep have more conserved residues among the different protein family members (Finn, et al., 2008). The most proximal annotated domain to the two mutations is the transmembrane domain, which demarks how the protein is situated in the plasma membrane of the cell. The locations of the p.E639K and p.S703N mutations fall in the cytoplasmic region of the protein, which is highly variable among the different species and family members and would probably affect protein interactions within the cell, potentially altering intracellular signaling.

Several cancer genome wide studies (Jones, et al., 2008; Sjoblom, et al., 2006; Wood, et al., 2007) clearly show that the cancer genetic landscape is made up of few genes that are frequently mutated (cancer 'mountain' genes) and a much larger number of genes that are infrequently mutated (cancer 'hill' genes) (Jones, et al., 2008). One main challenge is to decipher which of the discovered mutations have a functional role in cancer progression and are thus 'drivers' and which of the mutations are 'passengers'. While no direct inhibitors of ADAM genes have reached clinical development yet, targeting altered ADAM-mediated effector pathways has very recently entered clinical development: for example, targeting ADAM17-mediated ligand cleavage to inhibit Erb receptor signaling through inhibition of its secretase activity, or ADAM10 regulated Notch signaling are prime examples for such efforts. The novel gamma secretase inhibitor RO4929097 (Tolcher, et al., 2010) or the Notch inhibitor MK0752 (Deangelo, et al., 2010) have recently completed phase I clinical trials. As we find that several of the novel alterations have a functional effect on the protein suggesting that these are indeed drivers in melanoma, additional research on the role of the gene family in human cancers might discover novel therapeutic avenues.

\section{Supplementary Material}

Refer to Web version on PubMed Central for supplementary material.

\section{Acknowledgments}

We thank Dr. R. Weinberg for the Mel-STR cell line and Dr. W. Gahl and W. Westbroek (National Institutes of Health) for normal melanocytes RNA. We thank T. Prickett and V. Walia for their helpful comments on the manuscript. We also thank members of the NISC Comparative Sequencing Program for providing leadership in the generation of the sequence data analyzed here. Funded by the National Human Genome Research Institute, National Institutes of Health to Y.S. and Ministerio de Ciencia e Innovación-Spain, Fundación "M. Botín", and European Union (FP7 MicroEnviMet) to C.L-O.

\section{REFERENCES}

The Universal Protein Resource (UniProt) in 2010. Nucleic Acids Res. 38(Database issue):D142-8. [PubMed: 19843607]

Bhangale TR, Stephens M, Nickerson DA. Automating resequencing-based detection of insertiondeletion polymorphisms. Nat Genet. 2006; 38(12):1457-62. [PubMed: 17115056] 
Blobel CP. ADAMs: key components in EGFR signalling and development. Nat Rev Mol Cell Biol. 2005; 6(1):32-43. [PubMed: 15688065]

Bode W, Gomis-Ruth FX, Stockler W. Astacins, serralysins, snake venom and matrix metalloproteinases exhibit identical zinc-binding environments (HEXXHXXGXXH and Met-turn) and topologies and should be grouped into a common family, the 'metzincins'. FEBS Lett. 1993; 331(1-2):134-40. [PubMed: 8405391]

Dalgliesh GL, Furge K, Greenman C, Chen L, Bignell G, Butler A, Davies H, Edkins S, Hardy C, Latimer C, et al. Systematic sequencing of renal carcinoma reveals inactivation of histone modifying genes. Nature. 2010; 463(7279):360-3. [PubMed: 20054297]

Deangelo DJ, Stone RM, Silverman LB, Stock W, Attar EC, Fearen I, Dallob A, Matthews C, Stone J, Freedman SJ. A phase I clinical trial of the notch inhibitor MK-0752 in patients with T-cell acute lymphoblastic leukemia/lymphoma (T-ALL) and other leukemias. ASCO abstract. 2010:6585. others.

Edwards DR, Handsley MM, Pennington CJ. The ADAM metalloproteinases. Mol Aspects Med. 2008; 29(5):258-89. [PubMed: 18762209]

Finn RD, Tate J, Mistry J, Coggill PC, Sammut SJ, Hotz HR, Ceric G, Forslund K, Eddy SR, Sonnhammer EL. The Pfam protein families database. Nucleic Acids Res. 2008; 36(Database issue):D281-8. others. [PubMed: 18039703]

Gordon D, Abajian C, Green P. Consed: a graphical tool for sequence finishing. Genome Res. 1998; 8(3):195-202. [PubMed: 9521923]

Greenman C, Stephens P, Smith R, Dalgliesh GL, Hunter C, Bignell G, Davies H, Teague J, Butler A, Stevens C. Patterns of somatic mutation in human cancer genomes. Nature. 2007; 446(7132):1538. others. [PubMed: 17344846]

Gupta PB, Kuperwasser C, Brunet JP, Ramaswamy S, Kuo WL, Gray JW, Naber SP, Weinberg RA. The melanocyte differentiation program predisposes to metastasis after neoplastic transformation. Nat Genet. 2005; 37(10):1047-54. [PubMed: 16142232]

Hattori M, Osterfield M, Flanagan JG. Regulated cleavage of a contact-mediated axon repellent. Science. 2000; 289(5483):1360-5. [PubMed: 10958785]

Jemal A, Siegel R, Ward E, Hao Y, Xu J, Thun MJ. Cancer statistics, 2009. CA Cancer J Clin. 2009; 59(4):225-49. [PubMed: 19474385]

Jones S, Zhang X, Parsons DW, Lin JC, Leary RJ, Angenendt P, Mankoo P, Carter H, Kamiyama H, Jimeno A. Core signaling pathways in human pancreatic cancers revealed by global genomic analyses. Science. 2008; 321(5897):1801-6. others. [PubMed: 18772397]

Lopez-Otin C, Bond JS. Proteases: multifunctional enzymes in life and disease. J Biol Chem. 2008; 283(45):30433-7. [PubMed: 18650443]

Lopez-Otin C, Hunter T. The regulatory crosstalk between kinases and proteases in cancer. Nat Rev Cancer. 2010; 10(4):278-92. [PubMed: 20300104]

Ng PC, Henikoff S. SIFT: Predicting amino acid changes that affect protein function. Nucleic Acids Res. 2003; 31(13):3812-4. [PubMed: 12824425]

Palavalli LH, Prickett TD, Wunderlich JR, Wei X, Burrell AS, Porter-Gill P, Davis S, Wang C, Cronin JC, Agrawal NS. Analysis of the matrix metalloproteinase family reveals that MMP8 is often mutated in melanoma. Nat Genet. 2009; 41(5):518-20. others. [PubMed: 19330028]

Parsons DW, Jones S, Zhang X, Lin JC, Leary RJ, Angenendt P, Mankoo P, Carter H, Siu IM, Gallia GL. An integrated genomic analysis of human glioblastoma multiforme. Science. 2008; 321(5897):1807-12. others. [PubMed: 18772396]

Peschon JJ, Slack JL, Reddy P, Stocking KL, Sunnarborg SW, Lee DC, Russell WE, Castner BJ, Johnson RS, Fitzner JN. An essential role for ectodomain shedding in mammalian development. Science. 1998; 282(5392):1281-4. others. [PubMed: 9812885]

Pleasance ED, Cheetham RK, Stephens PJ, McBride DJ, Humphray SJ, Greenman CD, Varela I, Lin ML, Ordonez GR, Bignell GR. A comprehensive catalogue of somatic mutations from a human cancer genome. Nature. 2010; 463(7278):191-6. others. [PubMed: 20016485]

Prickett TD, Agrawal NS, Wei X, Yates KE, Lin JC, Wunderlich JR, Cronin JC, Cruz P, Rosenberg SA, Samuels Y. Analysis of the tyrosine kinome in melanoma reveals recurrent mutations in ERBB4. Nat Genet. 2009; 41(10):1127-32. [PubMed: 19718025] 
Qi H, Rand MD, Wu X, Sestan N, Wang W, Rakic P, Xu T, Artavanis-Tsakonas S. Processing of the notch ligand delta by the metalloprotease Kuzbanian. Science. 1999; 283(5398):91-4. [PubMed: 9872749]

Ruan J, Li H, Chen Z, Coghlan A, Coin LJ, Guo Y, Heriche JK, Hu Y, Kristiansen K, Li R. TreeFam: 2008 Update. Nucleic Acids Res. 2008; 36(Database issue):D735-40. others. [PubMed: 18056084]

Schlondorff J, Blobel CP. Metalloprotease-disintegrins: modular proteins capable of promoting cellcell interactions and triggering signals by protein-ectodomain shedding. J Cell Sci. 1999; 112(Pt 21):3603-17. [PubMed: 10523497]

Seals DF, Courtneidge SA. The ADAMs family of metalloproteases: multidomain proteins with multiple functions. Genes Dev. 2003; 17(1):7-30. [PubMed: 12514095]

Sjoblom T, Jones S, Wood LD, Parsons DW, Lin J, Barber TD, Mandelker D, Leary RJ, Ptak J, Silliman N. The consensus coding sequences of human breast and colorectal cancers. Science. 2006; 314(5797):268-74. others. [PubMed: 16959974]

Stocker W, Bode W. Structural features of a superfamily of zinc-endopeptidases: the metzincins. Curr Opin Struct Biol. 1995; 5(3):383-90. [PubMed: 7583637]

TCGA. Comprehensive genomic characterization defines human glioblastoma genes and core pathways. Nature. 2008; 455:1061-1068. [PubMed: 18772890]

Tolcher AW, Mikulski SM, Messersmith WA, Kwak EL, Gibbon D, Boylan J, Xu ZX, deMario M, Wheler JJ. A phase I study of RO4929097, a novel gamma secretase inhibitor, in patients with advanced solid tumors. ASCO abstract. 2010:2502.

Touab M, Villena J, Barranco C, Arumi-Uria M, Bassols A. Versican is differentially expressed in human melanoma and may play a role in tumor development. Am J Pathol. 2002; 160(2):549-57. [PubMed: 11839575]

Viloria CG, Obaya AJ, Moncada-Pazos A, Llamazares M, Astudillo A, Capella G, Cal S, Lopez-Otin C. Genetic inactivation of ADAMTS15 metalloprotease in human colorectal cancer. Cancer Res. 2009; 69(11):4926-34. [PubMed: 19458070]

Wood LD, Parsons DW, Jones S, Lin J, Sjoblom T, Leary RJ, Shen D, Boca SM, Barber T, Ptak J. The Genomic Landscapes of Human Breast and Colorectal Cancers. Science. 2007 others.

Yamagata M, Suzuki S, Akiyama SK, Yamada KM, Kimata K. Regulation of cell-substrate adhesion by proteoglycans immobilized on extracellular substrates. J Biol Chem. 1989; 264(14):8012-8. [PubMed: 2470739] 


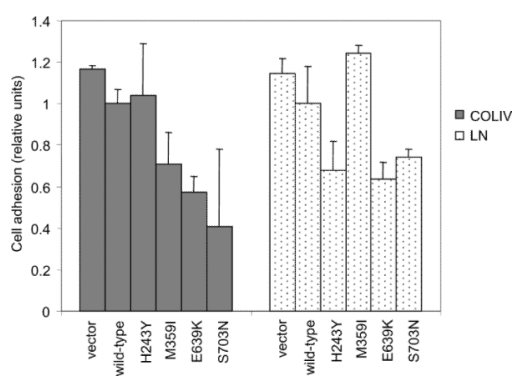

Figure 1.

Mutant ADAM7 modulates cell adhesion. Adhesion properties of Mel-STR cells overexpressing either the wild-type or mutant ADAM7 forms were assessed using the ECM Cell Adhesion Array kit. Collagen IV (COLIV), laminin (LN), $(n=2)$. 


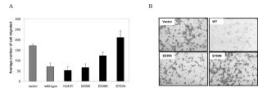

Figure 2.

Effects of ADAM7 mutation on cell migration. (A) Mel-STR cells overexpressing wild-type or mutant $A D A M 7$ were seeded in Boyden chambers and assessed for their migration abilities. Graph indicates the number of cells that migrated 24 hrs after seeding $(n=3)$. (B) Representative pictures of migrated cells. 


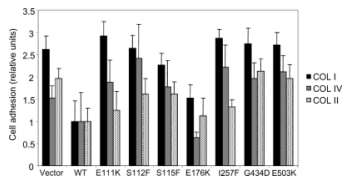

Figure 3.

Mutant ADAM29 modulates cell adhesion on collagen I, II and IV. Adhesion properties of A375 cells overexpressing either the wild-type or mutant ADAM29 forms were assessed with the ECM Cell Adhesion Array kit. collagen (COL), wild type (WT) $(n=2)$. 


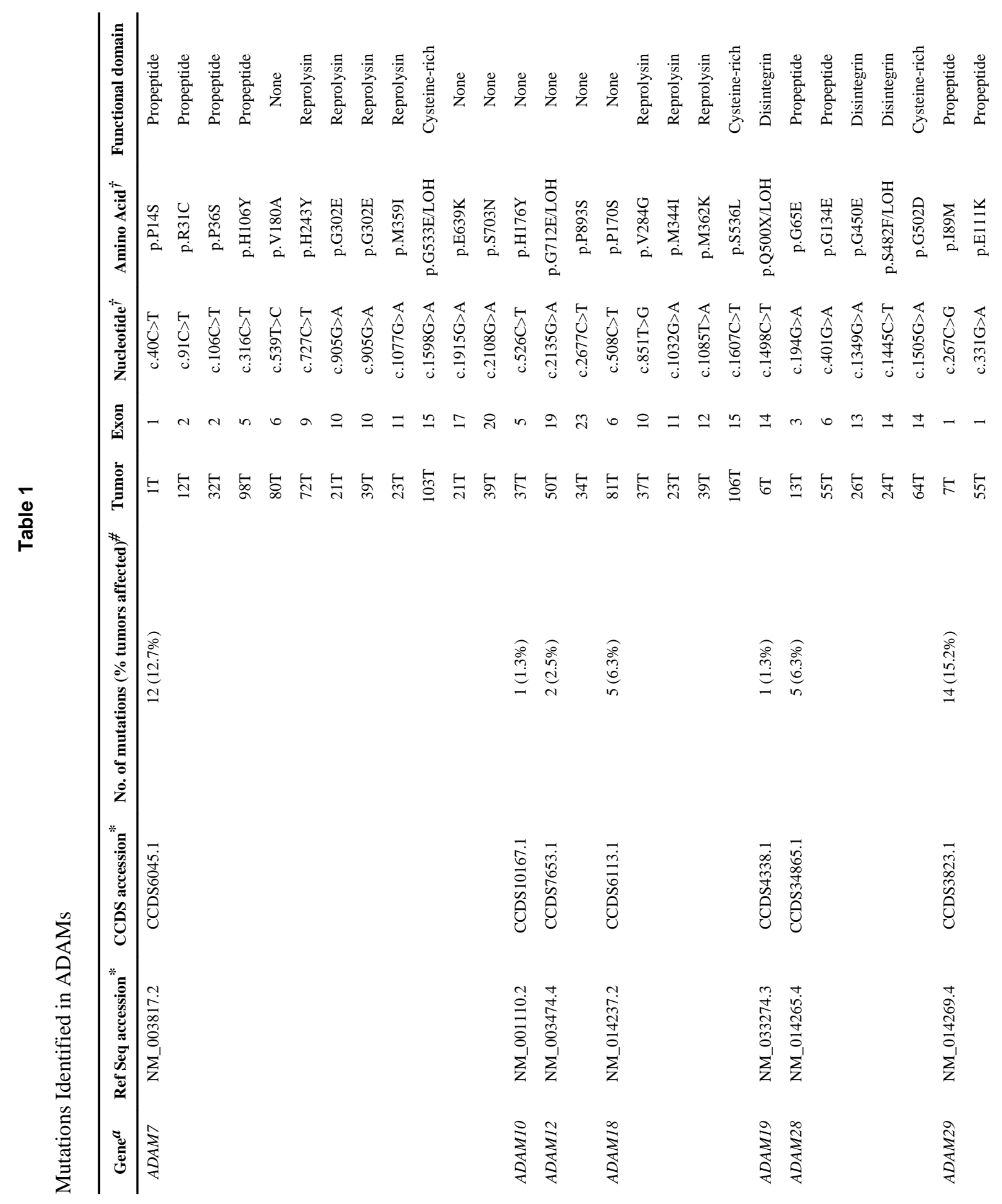




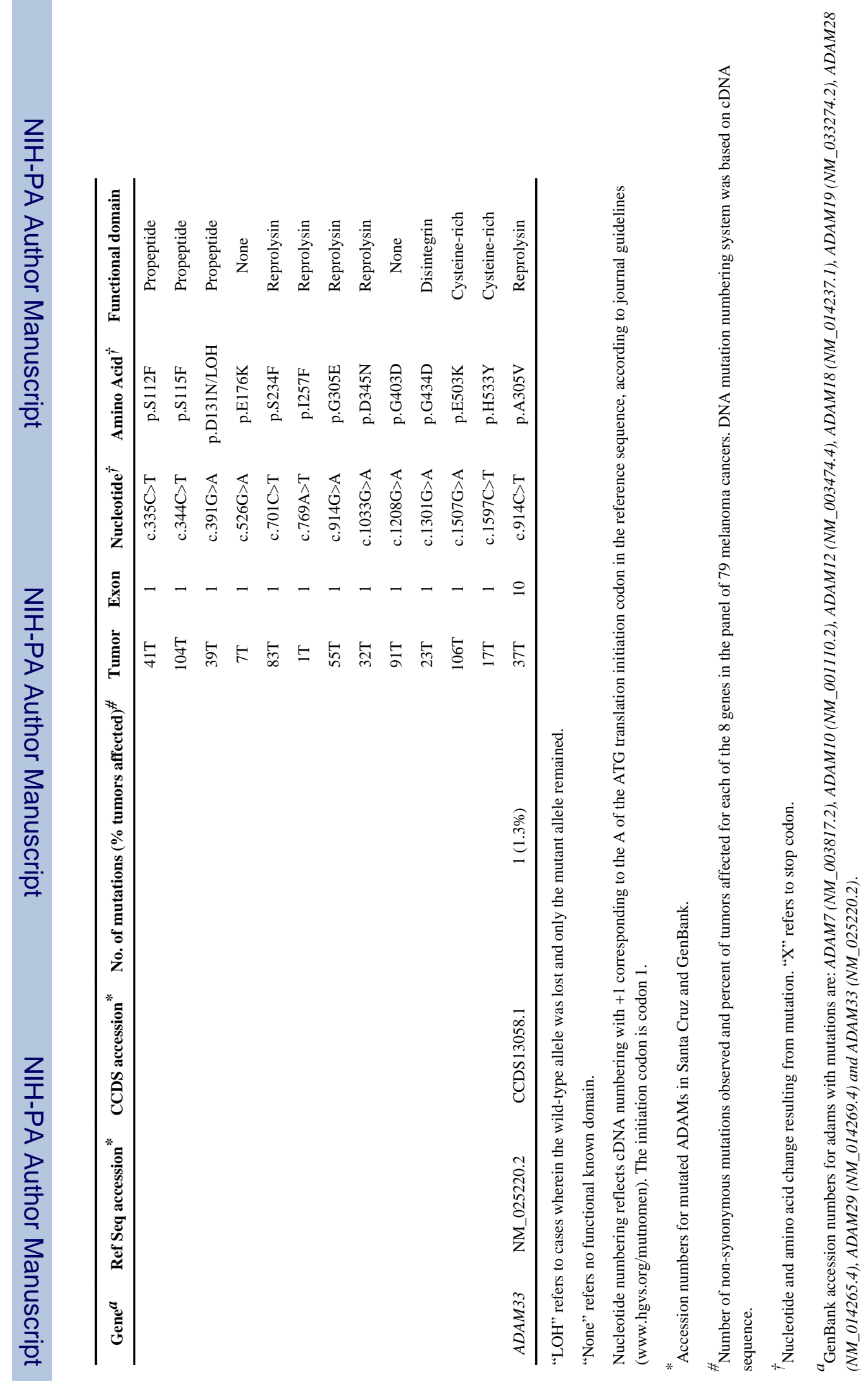


